\title{
O conteúdo da vida amorosa de estudantes universitários
}

\author{
Ailton Amélio da Silva \\ Universidade de São Paulo
}

\begin{abstract}
RESUMO
Embora existam muitas teorias e concepções sobre o amor, poucos estudos descreveram o conteúdo da vida amorosa. Esta pesquisa tem os seguintes objetivos: descrever as incidências de seis tipos de experiências amorosas; identificar as variabilidades destas experiências e suas inter-relações e verificar se há relação entre estas experiências e o sexo dos participantes. Participaram deste estudo 368 estudantes universitários: 303 na primeira amostra (177 mulheres e 126 homens) de três cursos universitários e 65 na segunda amostra (40 mulheres e 25 homens) de dois cursos universitários. A idade média destes estudantes era 24,3 anos (homens 23,9 anos e mulheres 24,7 anos). As incidências médias por participante das experiências amorosas foram as seguintes: "Ficar" - 26,1 parceiros; Parceiros Sexuais - 4,9; Namorados - 2,9; Paixões - 3,6; Amores - 1,3 e Percentagem de Participantes Apaixonados - 64\%. As distribuições de várias destas experiências dependeram do sexo dos participantes Várias das experiências amorosas das duas amostras apresentaram relações estaticamente significantes entre si o que indica uma grande similaridade entre elas.
\end{abstract}

Palavras-chave: relacionamento amoroso; experiências amorosas; amor; sexo

\begin{abstract}
The content of love relationships of college students

Although there are many theories and conceptions about love, few studies had described the content of the loving life. This research has the following objectives: describe the incidences of six types of loving experiences; identify the variabilities of these experiences and its inter-relations, and to verify if there's any relation between these experiences and the gender of the participants. Participants were 368 college students: 303 in the first sample (177 women and 126 men) from three university courses and 65 in the second sample ( 40 women and 25 men) from two university courses. The mean age of these students was 24,3 years (men 23,9 years and women 24,7 years). The average incidences of loving experiences for participant was: flirting $-26,1$ partners; sexual relationship $-4,9$; dating $-2,9$; passion $-3,6$; love $-1,3$ and percentage of participants in love $-64 \%$. The distributions of several of these experiences depended on the sex of the participants. Several of the loving experiences of the two samples presented statistically significant relations between them, indicating great similarities between these experiences.
\end{abstract}

Keywords: love relationship; love experience; love; sex

$\mathrm{O}$ relacionamento amoroso é freqüentemente abordado nas conversas, literatura, novelas, filmes e músicas. Um dos motivos da grande dedicação a este tema é que os acontecimentos nesta área têm implicações para a vida do indivíduo e para o futuro da humanidade. Segundo Darwin (1871), tão importante quanto sobreviver é procriar. Sobreviver diz respeito diretamente ao indivíduo. Procriar diz respeito à sobrevivência da humanidade.

A situação amorosa afeta praticamente todas as áreas da nossa vida. Existem estimativas de que mais de $90 \%$ das pessoas casam pelo menos uma vez
(Gottman \& Carrère, 1994). A maioria daqueles que não se casam também é afetada por este tipo de relacionamento: tem problemas psicológicos por não ter um parceiro, encontra obstáculos para exercer certos cargos, não é convidada para certos tipos de eventos, mulheres que têm casamentos conturbados e homens que se separam e não voltam a se casar têm mais chance de desenvolverem doenças orgânicas (Gottman \& Notarius, 2002). Segundo Seligman (2002), o casamento é um fator de felicidade mais potente que o trabalho, a situação financeira e a comunidade. 
Alguns aspectos do relacionamento amoroso têm sido objetos de pesquisas extensas, muitas vezes de âmbito nacional e até mesmo multicultural como, por exemplo, os requisitos que são importantes para a escolha de um cônjuge (Buss, 1994; Buss \& cols., 1990). A pesquisa de Kinsey, Pomeroy e Martin (1948) estabeleceu um marco nos estudos científicos da sexualidade. Posteriormente outros autores seguiram a linha de Kinsey. Por exemplo, Sharin Hite publicou alguns livros sobre os hábitos sexuais dos americanos que se tornaram fenômenos de venda (Hite, 1992). Estas pesquisas, no entanto, receberam muitas críticas devido a falhas na representatividade populacional das amostras estudadas (Michael, Gagnon, Laumann \& Kolata 1995). Mais recentemente, uma pesquisa muito cuidadosa sobre os hábitos sexuais dos americanos foi realizada por Michael e cols. (1995). Aqui no Brasil, o IBGE periodicamente apresenta dados sobre as incidências na população de solteiros, casados, viúvos, uniões consensuais, separados, desquitados, divorciados (IBGE, 2002). Recentemente Abdo, Moreira Jr e Fittipaldi (2000) realizaram uma pesquisa bastante ampla sobre os hábitos sexuais e incidências de disfunções em várias regiões do Brasil.

Uma outra linha de pesquisa que tem frutificado nesta área procura elucidar os mecanismos envolvidos em alguns dos conteúdos específicos da vida amorosa. Estas pesquisas procuram esclarecer a estrutura, as causas e os efeitos de cada um destes tipos de conteúdos e não as suas incidências, como as outras pesquisas citadas anteriormente. Alguns destes conteúdos mais pesquisados são: a sexualidade (Kaplan, 1977; Masters \& Johnson, 1984; Rhodes, Simmons \& Peters, 2005), os fatores que contribuem para a satisfação e a duração do casamento (Amélio \& Martinez, 2005; Pinsof, 2002; Stafford \& Kline, 2004) e o amor (Hendrick \& Hendrick, 1986; Lee, 1996; Silva \& cols., 2005; Shaver \& Hazan, 1998; Silva \& cols., 2005; Sternberg \& Barnes, 1988).

Embora o relacionamento amoroso tenha tanta importância, vários de seus aspectos ainda foram pouco estudados cientificamente. Por exemplo, existem poucos dados sobre as incidências de pessoas que estão sozinhas, namorando, apaixonadas, amando e sobre as quantidades de namorados, parceiros, parceiros sexuais, paixões e amores que ocorrem durante a vida.

Felizmente existem algumas exceções quanto à carência de estudos nesta área. Quatro destas exceções serão destacadas aqui. Uma delas é a pesquisa americana realizada por Michel e cols. (1995). Esta pesquisa, no entanto, não abordou práticas e sentimentos que são parte essencial do início, desenvolvimento e manutenção de um relacionamento amoroso, tais como, a incidência de pessoas que estão na fase do flerte ou namorando. Duas outras pesquisas merecem destaque porque apresentam dados sobre a incidência de pessoas apaixonadas. Uma pesquisa intercultural, realizada por Sprecher e cols. (1994), verificou que em três países, culturalmente muito diferentes entre si, a maioria das pessoas declarava que estava amando: $73 \%$ das russas e $61 \%$ dos russos, $63 \%$ dos japoneses e $41 \%$ das japonesas e $63 \%$ das americanas e $53 \%$ dos americanos declaram que estavam amando no momento que participaram dessa pesquisa. Outra pesquisa que estudou este fenômeno foi realizada por Hendrick e Hendrick (1986). Esses autores pediram a estudantes de duas universidades americanas que respondessem a um questionário sobre o amor. As percentagens daqueles estudantes dessas duas universidades que declararam que estavam amando naquele momento foram muito semelhantes: em média 54\% declararam que estavam amando naquele momento. Estas percentagens confirmam os achados de Sprecher e cols. (1994) sobre os EUA - a maioria dos pesquisados declarou que estavam amando. Estas duas pesquisa relatam outro fato semelhante: em todas as culturas estudadas a maioria daqueles que estão amando são mulheres.

A terceira exceção a ser destacada é a pesquisa de Abdo e cols. (2000). Embora esta pesquisa não tenha utilizado uma amostra representativa da população brasileira, ela é uma das mais amplas realizadas até agora no nosso país. Estes pesquisadores investigaram várias práticas sexuais que são consideradas "normais" (por exemplo, a quantidade de relações sexuais e a freqüência de orgasmo) e várias outras que são disfuncionais (por exemplo, homens que têm problemas de ereção e mulheres que têm dificuldades para atingir o orgasmo), na população brasileira. Estes pesquisadores, no entanto, deram pouca atenção para as práticas e sentimentos que constituem o conteúdo e que são o sustentáculo da vida afetiva e sexual. Por exemplo, eles não investigaram práticas e sentimentos, tão presentes na vida amorosa, como o "paquerar", o "ficar" e o amar.

Os atos amorosos também são regulados por normas sociais que podem diferir para homens e mulheres. Por exemplo, existem evidências de que a quantidade de parceiros sexuais é diferente para homens e mulheres, embora para uma população onde haja um equilíbrio no número de homens e mulheres isto seja estatisticamente impossível (Silva, 1996). De qualquer 
forma a importância do sexo dos participantes parece ser um forte determinante dos sentimentos e práticas amorosas e deve ser levada em conta em qualquer pesquisa deste tipo (Buss, 1994; Hendrick \& Hendrick, 1996).

Também existem evidências de que o conteúdo do relacionamento está mudando rapidamente. Por exemplo, o ficar, as práticas sexuais, a incidência da separação e do recasamento mudaram bastante nas últimas décadas. Estes fatores devem ser levados em conta quando se faz uma pesquisa deste tipo (Gregersen, 1983; Hunt, 1974). O exame de duas amostras coletadas após um intervalo de tempo contribui para o entendimento desta questão.

Informações sobre as incidências das situações e experiências amorosas e suas inter-relações são importantes por diversos motivos: podem servir como referencial para avaliar o desenvolvimento individual (permitem uma comparação entre o que está acontecendo com uma pessoa e com outros membros da cultura), indicam facilidades e dificuldades para o envolvimento em situações amorosas e ajudam a compreender e a tomar medidas quanto à evolução populacional e quanto aos distúrbios relacionados com a vida amorosa (Michael \& cols., 1995). Por exemplo, na área da avaliação do desenvolvimento físico as descrições das faixas de alturas e dos pesos "normais para cada idade" são úteis para avaliar se uma criança está se desenvolvendo de uma forma saudável. Analogamente, na área amorosa, quando uma pessoa nunca se apaixonou até os 24 anos isto indica que ela pode estar com algum problema de vinculação afetiva. Estas informações sobre as experiências e práticas que são consideradas "normais" para a idade, sexo e grupo social também costumam ser usadas pelos parentes, grupo social, pelo próprio indivíduo afetado e pelos psicólogos para raciocinar sobre o que está ocorrendo.

O exame das publicações nesta área mostra uma superênfase em alguns aspectos da vida amorosa tais como o amor, a sexualidade e as incidências dos estados civis e uma carência de estudos sobre outros aspectos que constituem boa parte do conteúdo deste tipo de relacionamento, tais como as incidências do ficar, do namorar, das paixões e do amor, suas interrelações e sobre os seus papéis na vida afetiva.

Esta pesquisa foi desenvolvida com o objetivo de contribuir para o conhecimento destes aspectos mais ignorados, mas tão centrais na vida amorosa das pessoas.

Interação em Psicologia, Curitiba, jul./dez. 2006, (10)2, p. 301-312

\section{OBJETIVOS DESTA PESQUISA}

(1) Descrever a incidência dos seguintes tipos de situações amorosas: Paquerando, Namorando, Coabitando, Casados e Sozinho ${ }^{1}$.

(2) Descrever a incidência das seguintes experiências amorosas: Quantidade de Parceiros de Ficada, Quantidade de Parceiros Sexuais, Quantidade de Parceiros de Namoro, Quantidade de Paixões, Quantidade de Amores que os participantes tiveram durante a vida e as Percentagens de Participantes que Declaram que estavam Amando ou Não-amando na ocasião que responderam ao questionário.

(3) Verificar se há relação entre as incidências das experiências amorosas enumeradas acima, duas a duas.

(4) Verificar se há relação entre o sexo dos participantes e as incidências dos relacionamentos e experiências amorosas.

\section{MÉTODO}

\section{Participantes}

Participaram deste estudo 368 estudantes universitários. Uma parte dos dados foi coletada em 2002 e a outra em 2005. A primeira coleta dos dados foi realizada com 303 estudantes (177 mulheres e 126 homens) de três cursos universitários diferentes. A segunda coleta dos dados foi realizada com 65 estudantes (40 mulheres e 25 homens) de dois cursos universitários. A idade média destes estudantes era 23,8 anos (homens $=23,5$ anos e mulheres 24,2 anos). Estes participantes foram escolhidos porque atendiam a dois critérios: (1) eram facilmente acessáveis pelos experimentadores e (2) tinham idade suficiente para terem passado por vários tipos de experiências amorosas.

Estes dados foram coletados por alunos especiais, mestrandos e doutorandos de um curso de pósgraduação ministrado por este autor ${ }^{2}$.

\section{Materiais}

Os tipos de situações e experiências amorosas incluídas neste estudo são aqueles mais mencionados em entrevistas informais realizadas com universitários por este autor.

O termo "situação amorosa", utilizado nesta pesquisa, para designar o tipo de relacionamento que existe entre duas pessoas engloba as situações legalmente denominadas de "estados civis" (solteiro, casado etc.) e outros tipos de situações cujos rótulos são usados corriqueiramente para nomear outros estágios 
de um relacionamento amoroso não previstos pela legislação atual ("ficando", namorando etc.). O termo "experiências amorosas" é utilizado aqui para nomear as práticas (ficar, fazer sexo etc.) e sentimentos (paixão, amor etc.) que geralmente ocorrem nos diversos tipos de relacionamentos amorosos.

As seguintes questões foram utilizadas na coleta dos dados desta pesquisa.

\section{Situações e experiências amorosas}

a) Informações demográficas

- Idade;

- Sexo (F/M);

b) Situação amorosa atual: sozinho, paquerando, namorando, morando com alguém, casado, outros; (aos respondentes, foi solicitado que indicassem número anos/meses da situação atual):

c) Experiências amorosas:

- número de parceiros (diferentes) que já ficou na vida;

- número de vezes que você já se apaixonou na vida;

- número de vezes que já se amou na vida;

- número de namorados que já teve na vida;

- atualmente você está amando alguém?;

- número de parceiros sexuais que já teve na vida (excluir prostitutas).

Optou-se por não definir estes termos, uma vez que eles são de uso corrente. Enquetes preliminares, realizadas por este autor, com diversos grupos de estudantes indicaram que estes termos são usados fidedignamente por eles para classificar vários tipos de exemplos de práticas e sentimentos amorosos.

Os participantes não se identificaram em nenhum destes questionários garantindo assim o sigilo de seus resultados individuais e a maior credibilidade das respostas.

\section{Procedimento}

Todos os dados foram coletados através de aplicações coletivas durante o período de aula. Após a coleta, os participantes computaram os seus resultados individuais sobre os estilos de amor. Em seguida, os pesquisadores ministraram uma palestra sobre os significados destes estilos e dos escores individuais que haviam sido computados.

\section{RESULTADOS E DISCUSSÃO}

\section{Incidências das situações amorosas}

$\mathrm{O}$ tipo de situação amorosa mais freqüente para o conjunto de todos os participantes foi o Namorando $(40,5 \%)$. As percentagens dos outros tipos de tipos de situações amorosas, em ordem decrescente de incidência, foram: Casado $(15,7 \%)$, Paquerando $(14,3 \%)$, Outros $(5,4 \%)$ e Morando com alguém $(2,7 \%)$. O relacionamento amoroso está presente na vida da grande maioria dos participantes desta pesquisa. Apenas $22,2 \%$ deles se declararam Sozinhos. A grande maioria, $72,6 \%$, declarou que estava envolvida em algum tipo de relacionamento amoroso e 5,1\% optaram pela categoria Outros Tipos. Quando se considera um intervalo de tempo, e não apenas um dado momento como este captado pela pesquisa, parece provável que uma percentagem ainda maior de pessoas se envolva em relacionamentos amorosos: uma vez que os tipos de relacionamentos amorosos iniciais são relativamente instáveis, é possível imaginar que uma boa parte daqueles que se declararam Sozinhos, no momento que responderam esta pesquisa, rapidamente possam mudar para as situações Paquerando ou Namorando e vice-versa.

\section{Incidências e inter-relações entre as experiências amorosas}

Nesta sessão serão analisados os efeitos do sexo dos participantes nas frequiências de incidência de cada uma das experiências amorosas. Também serão analisadas as variabilidades destas incidências entre os participantes de cada uma destas experiências amorosas. Para analisar esta variabilidade, foram usados dois tipos de recursos: (1) seus desvios padrões e (2) as percentagens de incidências em cada quantidade ou faixas de quantidades de experiência.

Para construir as tabelas que mostram as variações das incidências de experiências amorosas entre os participantes da pesquisa, estes foram ordenados de acordo com a quantidade de cada um dos tipos de experiência que tiveram e, em seguida, foram categorizados segundo tais quantidades, ou faixas de quantidades. Estas quantidades de experiências amorosas são mostradas na primeira linha de cada tabela apresentada nesta seção. Em alguns casos, para facilitar a análise dos resultados, foram definidas faixas de quantidades de experiências amorosas com diferentes amplitudes. As escolhas destas amplitudes obedeceram pelo menos um dos três seguintes critérios: (1) 
interesse teórico por uma dada faixa (por exemplo, parece importante saber a percentagem de pessoas que nunca ficou. Por isso, foi definida a categoria "zero ficada"); (2) abranger uma percentagem nem muito pequena e nem muito grande de participantes (definidas aqui, arbitrariamente, como mais que $5 \%$ em pelo menos uma das subamostras e menos que $30 \%$ em todas as subamostras da faixa, respectivamente), (3) sempre que não contrariava os dois critérios anteriores, cada faixa deveria ter a mesma amplitude que outras da mesma tabela.

A categorização por Ano da Coleta dos Dados não será apresentada aqui e nas descrições dos outros tipos de experiências amorosas porque elas são muito semelhantes às categorizações por sexo.

\section{Ficar}

A quantidade média de parceiros de ficada para o total de participantes desta pesquisa é $26,1(\sigma=34,6)$. A quantidade média de parceiros de ficada da subamostra de mulheres é 23,0 e da subamostra de homens 29,3 parceiros. A quantidade média de parceiros de ficada da subamostra de 2002 é 23,4 parceiros e da subamostra de 2005 28,9 parceiros.

Esta semelhança entre homens e mulheres nas Quantidades de Parceiros de Ficada era esperada com base no seguinte raciocínio: na população as quantidades de homens e mulheres são semelhantes. Quando um homem fica com uma mulher esta também fica com um homem. Portanto, as quantidades médias de Parceiros de Ficada de ambos os sexos devem ser semelhantes. Isto não implica que as incidências por faixa de quantidade de parceiros sejam iguais para ambos os sexos. Por exemplo, em um grupo de 10 homens e 10 mulheres, um só homem poderia ficar com as dez mulheres e os outros com nenhuma. Neste caso, a média para os homens e para as mulheres será de um parceiro de ficada, mas suas distribuições serão muito diferentes.

As quantidades médias de parceiros de ficada são muito semelhantes em três das quatro subamostras (na casa de 23 parceiros). Apenas na subamostra de Homens - 2005 esta quantidade (34,8 parceiros) discrepa das outras três subamostras. As semelhanças entre as incidências desta experiência amorosa entre os participantes de 2002 e 2005 também eram esperadas pelos seguintes motivos: são poucos os anos que separam estas duas coletas e há vários tipos de semelhanças entre as características dos participantes que podem influenciar este tipo de experiência. Por exemplo, suas idades e seus níveis socioeconômicos são semelhantes.

A média dos desvios padrões destas quatro subamostras é 34,5 parceiros de ficada. Os desvios padrões das quatro subamostras variaram entre 29,2 (Mulheres - 2002) e 46,2 (Homens - 2005). As quantidades individuais de parceiros de ficada variaram entre zero e 200. Esta grande variação entre os participantes e as magnitudes destes desvios padrões mostram que há uma grande dose de variação nas quantidades de parceiros de ficada.

Uma outra forma de avaliar a variação na Quantidade de Parceiros de Ficada é agrupar os participantes por faixas de Quantidades de Parceiros de Ficada que já tiveram durante a vida. Esta categorização, diferenciada por sexo, é apresentada na Tabela 1.

Tabela 1. Percentagens de participantes que tiveram cada uma das quantidades de parceiros de ficada especificada em cada uma das faixas

\begin{tabular}{cccccccc}
\hline Sexo dos & \multicolumn{7}{c}{ Quantidade de Parceiros de Ficadas (Faixas) } \\
\cline { 2 - 8 } Participantes & 0 & 1 a 5 & 6 a 10 & 11 a 20 & 21 a 50 & 51 a 80 & + que 81 \\
\hline Homens & 6,7 & 25,6 & 18,9 & 16,6 & 20,5 & 4,8 & 6,7 \\
Mulher & 5,2 & 22,9 & 19,7 & 20,6 & 14,0 & 3,4 & 14,0 \\
\%s - Médias & 5,9 & 24,2 & 19,3 & 18,6 & 17,2 & 4,1 & 10,3 \\
\hline
\end{tabular}

A correlação entre as percentagens de incidência de homens e de mulheres nestas faixas foi de $r=0,89$ ( $5 \mathrm{gl}, \mathrm{p}<0,01)$. Esta correlação indica o grau de similaridade nas percentagens de participantes que ficaram cada uma das quantidades de vezes especificadas nesta tabela. A correlação entre as percentagens de incidência nas faixas de quantidades de parceiros de ficadas para as subamostras de 2002 e as de 2005 foi de 0,67 (5 gl, p>0,05). Um motivo para esta correlação não ter atingido a zona de significância é a pequena quantidade de graus de liberdade.

A última linha da Tabela 1 mostra as percentagens de todos os participantes da pesquisa que incidiram em cada uma das sete faixas de números de ficadas. Apenas 5,9\% dos participantes incidiram na primeira faixa - nunca ficaram (0 parceiro de ficada). Portanto, 
a grande maioria das pessoas destas amostras $(94,1 \%)$ já ficou com pelo menos um parceiro. As três faixas seguintes incluem as maiores percentagens de participantes: faixa de uma a cinco parceiros $(24,2 \%)$, faixa de 6 a 10 parceiros $(19,3 \%)$, faixa de 11 a 20 parceiros $(18,6 \%)$. Estas três percentagens são semelhantes entre si. A soma delas perfaz um total de $62,1 \%$ dos participantes. Ou seja, a maioria dos participantes teve de 1 a 20 parceiros. Embora as três últimas faixas desta tabela tenham amplitudes maiores do que as anteriores, as percentagens de participantes que nelas incidiram não são maiores do que as das outras faixas. Ou seja, poucos participantes tiveram grandes quantidades de parceiros de ficada. Por exemplo, apenas $10,3 \%$ dos participantes que tiveram mais que 81 parceiros.

\section{Ficar - relações com outros tipos de experiências amorosas}

Tanto para mulheres como para homens a Quantidade de Parceiros de Ficada está diretamente relacionada com o número de parceiros sexuais $(\mathrm{r}=0,44$ e $\mathrm{r}=$ 0,41 , respectivamente; 136 e $103 \mathrm{gls}$, respectivamente e $\mathrm{p}<0,01$, para ambas as correlações).

Para as mulheres a Quantidade de Parceiros de Ficada também está diretamente relacionada com a Idade $(\mathrm{r}=0,27 ; 136 \mathrm{gl}, \mathrm{p}<0,01)$ e com número de namorados $(r=0,32 ; 127 \mathrm{gl}, \mathrm{p}<0,01)$. O Ficar, portanto, está mais correlacionado com outras experiências amorosas para as mulheres do que para os homens. Uma hipótese para explicar este fenômeno é que elas são mais seletivas que eles na escolha dos parceiros de ficada: para elas estes também devem ter qualificações para serem namorados. Para eles, este segundo requisito parece ser menos necessário. A correlação negativa entre idade das mulheres e Quantidade de Parceiros de Ficada indica que as mais jovens estão ficando com mais parceiros do que as mais velhas. Uma possível explicação para isso é que esta prática ainda não estava bem estabelecida na época que estas mulheres estavam na idade de ficar e agora talvez não se sintam bem ou estejam impedidas de praticá-la porque já estão comprometidas (casadas ou morando com alguém, por exemplo).

\section{Quantidade de Parceiros Sexuais}

$\mathrm{O}$ número médio de parceiros sexuais para o total de participantes desta pesquisa é 5,6 $(\sigma=5,8)$. Os homens, em média, relataram, aproximadamente, o dobro de parceiros sexuais do que as mulheres (3,2 e 6,6 parceiros, respectivamente). Este quantidade bem maior de parceiros sexuais declarada pelos homens já foi relatada em outras pesquisas (Silva, 1996), inclusive em algumas que estudaram amostras representativas da população de todo um pais (por exemplo, Michael \& cols., 1995). Tal diferença é matematicamente impossível naqueles países onde as quantidades de homens e mulheres são semelhantes, pois cada vez que um homem pratica sexo com uma mulher esta faz o mesmo com ele e as médias de ambos os sexos são afetadas da mesma forma. Uma explicação possível para este fenômeno é que os homens exageram e as mulheres diminuem suas quantidades de parceiros sexuais. Em um pequeno grupo ou em certas idades este fenômeno é possível porque seus participantes podem praticar sexo com pessoas de outros grupos.

A média dos desvios padrões das quantidades de parceiros sexuais das quatro subamostras é de 5,8 parceiros. Estes desvios padrões variaram entre 3,6 (Mulheres - 2002) e 7,7 (Homens - 2005). As quantidades individuais de parceiros sexuais variaram entre 0 e 30 parceiros. Houve, portanto uma grande dose de variação nas quantidades de parceiros sexuais entre os participantes desta pesquisa.

Uma outra forma de avaliar a variação na quantidade de parceiros sexuais é agrupar os participantes por faixas de quantidades de parceiros sexuais que já tiveram durante a vida. Esta categorização para homens e mulheres é apresentada na Tabela 2.

Tabela 2. Percentagens de participantes que tiveram cada uma das quantidades de parceiros sexuais especificados na linha marginal desta tabela

\begin{tabular}{ccccccccccc}
\hline Sexo dos & \multicolumn{10}{c}{ Quantidades de parceiros sexuais } \\
\cline { 2 - 12 } Participantes & 0 & 1 & 2 & 3 & 4 & 5 & 6 & 7 a 10 & 11 a 19 & 20 ou + \\
\hline Mulheres & 11,3 & 35,3 & 18,7 & 11,2 & 4,0 & 5,3 & 0,9 & 7,2 & 2,8 & 2,5 \\
Homens & 5,5 & 15,3 & 15,6 & 10,2 & 3,5 & 7,5 & 8,7 & 17,6 & 3,1 & 12,7 \\
Médias & 8,4 & 25,3 & 17,1 & 10,6 & 3,7 & 6,4 & 4,8 & 12,4 & 2,9 & 7,6 \\
\hline
\end{tabular}

Não houve relação estatisticamente significante entre as percentagens de incidência de homens e de mulheres nas faixas de quantidades de parceiros sexuais $(\mathrm{r}=0,45 ; 9 \mathrm{gl} ; \mathrm{p}>0,05)$. Exemplos das dissimilaridades 
entre mulheres e homens: $11,3 \%$ das mulheres e 5,5\% dos homens eram virgens. 46,6\% das mulheres só tiveram 0 ou 1 parceiros sexuais. Apenas 20,8\% dos homens tiveram esta mesma quantia de parceiros sexuais. $5,3 \%$ das mulheres tiveram 11 ou mais parceiros; $15,6 \%$ dos homens tiveram esta quantidade de parceiros.

Houve relação estaticamente significante entre as percentagens de incidência nas faixas de quantidades de parceiros sexuais para as subamostras de 2002 e as de 2005 ( $\mathrm{r}=0,93,5 \mathrm{gl}, \mathrm{p}>0,05)$. Portanto estas duas amostras foram muito semelhantes neste tipo de incidência, o que indica a possibilidade de generalizar estas conclusões para outros grupos assemelhados a estes.

A última linha da Tabela 2 mostra as percentagens de todos os participantes da pesquisa cujos números de parceiros sexuais incidiram em cada uma das quantidades ou faixas de quantidades de parceiros sexuais. A primeira faixa, 0 parceiro, teve uma incidência de $8,4 \%$ dos participantes. Esta é a percentagens de virgens desta amostra. As maiores percentagens de participantes incidiram nas três quantidades seguintes de parceiros sexuais: um parceiro sexual (25,3\% dos participantes), 2 parceiros $(17,1 \%$ dos participantes), 3 parceiros (10,6\% dos participantes). A soma destas três percentagens perfaz um total de $48,0 \%$ dos participantes. Ou seja, aproximadamente metade dos participantes tiveram de 1 a 3 parceiros sexuais. $88,7 \%$ dos participantes tiveram no máximo 10 parceiros sexuais. Entrevistas realizadas pelo autor desta pesquisa indicam que a grande maioria dos parceiros sexuais é constituída por namorados e não por parceiros casuais. Estas quantidades de parceiros e a situação amorosa que existe entre eles dão uma idéia bem diferente daquela que seria esperada caso o sexo realmente estivesse liberado entre os jovens. Estes números são condizentes com os relatados na pesquisa de âmbito nacional (americana) realizada por Michael e cols. (1995). Por exemplo, nesta pesquisa apenas $15,1 \%$ dos homens e $2,7 \%$ das mulheres relataram que tiveram mais que 21 parceiros sexuais durante a vida.

\section{Quantidade de parceiros sexuais: relações com outros tipos de experiências amorosas}

Para homens e mulheres a Quantidade de Parceiros Sexuais está correlacionada com a Quantidade de Vezes que Ficou ( $r=0,44$ e 0,41, respectivamente; 138 e 102 gls, respectivamente; $p<0,01$ para ambos os casos) e com a Quantidade de Namorados ( $\mathrm{r}=0,40$ e $\mathrm{r}=0,42$, respectivamente; 128 e $107 \mathrm{gls}$, respectivamente; $\mathrm{p}<0,01$ para ambos os casos). Para as mulheres a Quantidade de Parceiros Sexuais está correlacionada com a Quantidade de Vezes que se Apaixonou $(\mathrm{r}=0,22,141 \mathrm{gls}, \mathrm{p}<0,05)$. Para os homens a Quantidade de Parceiros Sexuais está correlacionada com a idade $(\mathrm{r}=0,42,101 \mathrm{gl}, \mathrm{p}<0,01)$. No caso desta pratica amorosa aconteceu o que era previsto pelas teorias psicobiológicas: a Quantidade de Parceiros Sexuais para as mulheres está mais associada com os sentimentos amorosos (Paixão) e para homens com as oportunidades propiciadas pelo Ficar, Namorar e mais anos de vida (Buss, 1994).

\section{Quantidade de Namorados}

A média de namorados por participante da pesquisa foi $2,9(\sigma=2,1)$. As quantidades médias de namorados das mulheres foi 3,0) e dos homens 2,7 namorados.

A média dos desvios padrões das quantidades de namorados das quatro subamostras é de 2,1 parceiros. Os desvios padrões das médias de namorados das quatro subamostras variaram entre 1,9 (Mulheres 2005) e 2,6 (Homens - 2002) As quantidades individuais de namorados variaram entre 0 e 10 . As magnitudes destes desvios padrões revelam uma boa dose de variação nas quantidades de namorados entre os participantes desta pesquisa.

Uma outra forma de avaliar a variação na quantidade de namorados é agrupar os participantes por faixas de quantidades de apaixonamentos que já tiveram durante a vida. Esta categorização para homens e mulheres é apresentada na Tabela 3.

Tabela 3. Percentagens de participantes que tiveram cada uma das quantidades de namorados especificadas na linha de entrada desta tabela

\begin{tabular}{ccccccccc}
\hline Sexo dos Participantes & \multicolumn{7}{c}{ Quantidade de namorados } \\
\cline { 2 - 10 } & 0 & 1 & 2 & 3 & 4 & 5 & 6 & 7 ou mais \\
\hline Mulheres & 3,6 & 13,9 & 27,0 & 26,4 & 12,4 & 5,3 & 5,4 & 5,8 \\
Homens & 13,1 & 23,8 & 17,7 & 21,3 & 5,5 & 7,8 & 3,9 & 6,7 \\
$\%$ - MÉDIA & 8,3 & 18,8 & 22,3 & 23,8 & 8,9 & 6,5 & 4,6 & 6,2 \\
\hline
\end{tabular}


É raro ter tido zero ou mais que sete namorados. Apenas 5,5\% dos participantes tiveram zero namorado e apenas $7,8 \%$ tiveram sete ou mais namorados. Ou seja, $86,7 \%$ dos participantes tiveram de 1 a 6 namorados e $70,9 \%$ dos participantes tiveram de 1 a 4 namorados.

A correlação entre as percentagens de incidência de homens e de mulheres nestas faixas de quantidades de namorados foi de $\mathrm{r}=0,75$ ( $6 \mathrm{gls}, \mathrm{p}<0,01)$. Esta correlação era esperada uma vez que homens namoram mulheres e vice-versa e, portanto, seus padrões devem ser semelhantes. A correlação entre as percentagens de incidência nas faixas de quantidades de parceiros de ficadas para as subamostras de 2002 e as de 2005 foi de 0,81 (5 gl, p>0,05). Esta correlação indica o grau de similaridade entre estas duas subamostras neste tipo de distribuição e a possibilidade de generalização destes resultados para outras amostras similares.

Como geralmente homem namora mulher e viceversa, como conciliar o fato das correlações não serem muito altas? Para responder esta questão é necessário admitir que as práticas de ambos os sexos são complementares, mas podem diferir nas suas distribuições. Por exemplo, mais homens do que mulheres podem ter muitos namorados. Caso isto aconteça, um outro grupo de homens vai ter que compensar aquele grupo (haveria um equilíbrio entre os sexos, em média, mas descompensações em determinadas faixas etárias ou grupos).

\section{Quantidade de Namorados: relações com outras experiências amorosas}

Tanto para mulheres como para homens a Quantidade de Namorados está diretamente relacionada com a idade, $(r=0,32$ e $r=0,41$ respectivamente; 133 e 114 gls, respectivamente; $p<0,01$ para ambos os casos), com a Quantidade de Paixões ( $r=0,33$ e $r=0,21$ respectivamente; 140 e 112 gls respectivamente; $p<0,01$ para ambos os casos) e com a Quantidade de Parceiros Sexuais $(r=0,40$ e $r=0,42$, respectivamente; 127 e 103 gls respectivamente; $\mathrm{p}<0,01$ para ambos os casos).
Todas estas relações eram esperadas, uma vez que estas experiências amorosas são consideras como desejáveis para o namoro e vice-versa. Para as mulheres a quantidade de namorados está diretamente relacionada com a Quantidade de Vezes que Ficou $(\mathrm{r}=0,34,139 \mathrm{gl}, \mathrm{p}<0,01)$. $\mathrm{O}$ fato de haver relação entre namorar e ficar apenas para as mulheres parece sugerir que elas são mais seletivas com aqueles que ficam - os parceiros de ficada também teriam qualidades para serem namorados. Os homens, segundo esta hipótese, seriam menos exigentes e mais oportunísticos (Buss, 1994). Para os homens a quantidade de namoradas está inversamente relacionada com o fato de estar amando ( $\mathrm{r}=-0,29,111 \mathrm{gl}, \mathrm{p}<0,01)$. Esta relação talvez sugira que aqueles homens mais capazes de amar têm menos namoradas porque ficam mais tempos comprometidos com aquelas que amam.

\section{Apaixonar}

A média de apaixonamentos para todos os participantes da pesquisa foi de 3,6 vezes. Em média as mulheres se apaixonaram 4,2 vezes e os homens 3,1 vezes.

As médias de apaixonamentos das subamostras variaram entre 2,9 vezes (homens da amostra de 2002) e 4,2 vezes (mulheres das amostras de 2002 e 2005). A média dos desvios padrões das quatro subamostras é de 4,4 apaixonamentos. Os desvios padrões das Quantidades de Apaixonamentos das quatro subamostras variaram entre 2,6 (Homens - 2002) e 7,8 (Mulheres 2005). As quantidades individuais de apaixonamentos variaram entre zero e 20 paixões. As magnitudes destes desvios padrões revelam haver uma grande dose de variação nas quantidades de apaixonamentos entre os participantes desta pesquisa.

Uma outra forma de avaliar a variação na quantidade de apaixonamentos é agrupar os participantes por faixas de quantidades de apaixonamentos que já tiveram durante a vida. Esta categorização para homens e mulheres é apresentada na Tabela 4.

Tabela 4. Percentagens de participantes que tiveram cada uma das quantidades de paixões especificadas na linha marginal desta tabela

\begin{tabular}{ccccccccc}
\hline Sexo dos & \multicolumn{7}{c}{ Quantidade de paixões } \\
\cline { 2 - 10 } Participantes & 0 & 1 & 2 & 3 & 4 & 5 & 6 & 7 ou + \\
\hline Mulheres & 3,3 & 14,5 & 29,0 & 18,6 & 10,9 & 9,4 & 3,7 & 10,4 \\
Homens & 5,1 & 16,7 & 33,3 & 17,5 & 10,1 & 4,6 & 1,1 & 9,4 \\
Médias & 4,2 & 15,6 & 31,1 & 18,0 & 10,5 & 7,0 & 2,4 & 9,9 \\
\hline
\end{tabular}


A Tabela 4 mostra as percentagens de homens e mulheres que tiveram cada uma das quantidades de apaixonamentos especificadas na primeira linha.

A correlação entre as percentagens de incidência de homens e percentagem de mulheres nas faixas de quantidades de apaixonamentos foi $\mathrm{r}=0,91$ (5 gl, $\mathrm{p}<0,01)$. A correlação entre as distribuições deste mesmo tipo de percentagens nas subamostras de 2002 e 2004 foi também 0,91 ( $5 \mathrm{gl}, \mathrm{p}<0,01)$. Portanto houve uma forte similaridade nas percentagens de incidências de apaixonamentos de homens e mulheres e nas percentagens de incidências das amostras de 2002 e 2005.

Uma vez que a média de apaixonamentos das mulheres parece ser maior do que a dos homens e o padrão de distribuição das percentagens de participantes nas quantidades de apaixonamentos não depende do sexo, isto aponta uma tendência para haver mais mulheres apaixonadas do que homens na maioria das faixas de quantidades de apaixonamentos.

A quantidade de apaixonamentos onde se verificou a maior incidência de participantes $(31 \%)$ foi a de duas vezes. A grande maioria dos participantes $(75,2 \%)$ se apaixonou de uma a quatro vezes. Nunca ter se apaixonado ( $4,2 \%$ dos participantes) ou ter se apaixonado sete ou mais vezes $(9,9 \%)$ aconteceu raramente entre os participantes desta pesquisa (soma das percentagens nos dois casos $=14,1 \%$ dos participantes).

Quantidade de apaixonamentos - relações com outros tipos de experiências amorosas

Tanto para as mulheres como para os homens a Quantidade de Apaixonamentos está correlacionada com a Quantidade de Vezes que Amou ( $\mathrm{r}=0,35$ e $\mathrm{r}=0,38$, respectivamente; 142 e 112 gls, respectivamente, $\mathrm{p}<0,01$ para ambos os casos) e Quantidade de Namorados ( $\mathrm{r}=0,33$ e $\mathrm{r}=0,21$, respectivamente; $134 \mathrm{e}$ $113 \mathrm{gls}$, respectivamente $\mathrm{p}<0,01$ e $\mathrm{p}<0,05$, respectivamente). Para as mulheres a quantidade de apaixonamentos também está positivamente associada com a quantidade de parceiros sexuais $(\mathrm{r}=0,22,121 \mathrm{gls}$, $\mathrm{p}<0,05)$.

Esta é a variável que apresenta o maior número de relações com as outras experiências amorosas (cinco correlações estatisticamente significantes). A correlação entre as Quantidades de Vezes que Amou com a Quantidade de Namorados tanto para homens como para mulheres indica que apaixonar pode ser um primeiro passo para iniciar um namoro. Por outro lado, um levantamento que fizemos indicou que quase a metade dos namoros é iniciada sem paixão. Neste caso as pessoas tendem a se apaixonar por quem estão namorando (isto poderia ser explicado pela teoria da atribuição). Apaixonar também pode ser um primeiro estágio para o amor, como indicado pela correlação entre estes dois tipos de experiências: uma parte daqueles fatores que levariam uma pessoa a se apaixonar pela outra também faria que ela amasse a outra, caso outros requisitos fossem preenchidos.

\section{Quantidade de vezes que amou}

Estes dados só foram coletados para a subamostra de 2002.

Estes participantes amaram, em média, 1,3 vez $(\sigma=1,0)$. Os números médios de vezes que mulheres e homens amaram foram 1,3 e 1,4 vez, respectivamente.

A média dos desvios padrões das duas subamostras é 1,1 parceiros $(\sigma=0,93$ amores para a subamostra de Mulheres - 2005 e $\sigma=1,2$ amores para a subamostra de Homens - 2002). As quantidades individuais de amores variaram entre zero e sete amores. As magnitudes destes desvios padrões revelam haver uma pequena dose de variação nas quantidades de amores entre os participantes desta pesquisa.

Uma outra forma de avaliar a variação na quantidade de amores é agrupar os participantes por faixas de quantidades de amores que já tiveram durante a vida. Esta categorização para homens e mulheres é apresentada na Tabela 5.

Tabela 5. Percentagens de participantes que amaram cada uma das quantidades de vezes (subamostra de 2002)

\begin{tabular}{ccccccc}
\hline Sexo dos & \multicolumn{5}{c}{ Quantidade de Amores } \\
\cline { 2 - 7 } Participantes & 0 & 1 & 2 & 3 & 4 & +4 \\
Mulheres & 7,9 & 64,0 & 21,3 & 5,1 & 0,6 & 1,1 \\
Homens & 17,4 & 52,3 & 18,2 & 6,1 & 1,5 & 4,5 \\
Médias & 12,6 & 58,1 & 19,7 & 5,6 & 1,0 & 2,8 \\
\hline
\end{tabular}


A Tabela 5 apresenta as percentagens de participantes que amaram cada uma das quantidades de vezes especificadas na primeira linha desta tabela. A inspeção visual desta tabela sugere que existem mais homens do que mulheres que amam poucas e muitas vezes (ver as percentagens na Tabela 8). Apesar desta diferença, o que prevalece é a semelhança nas incidências das percentagens de participantes nas quantidades de amores dos homens e mulheres $(\mathrm{r}=0,98$, 5 gls, $\mathrm{p}<0,01)$.

A quantidade de amores mais comum entre os participantes desta pesquisa foi uma vez $(58,1 \%$ dos participantes). A segunda quantidade mais freqüente foi duas vezes (19,7\% dos participantes). Apenas 12,6\% dos participantes declararam nunca ter amado (zero vez) e apenas 3,8\% amaram quatro ou mais vezes.

\section{Quantidade de vezes que amou - relações com outros tipos de experiências amorosas}

Tanto para mulheres como para homens a Quantidade de Vezes que Amou está diretamente relacionada com a Quantidade de Vezes que se Apaixonou ( $r=0,35$ e $\mathrm{r}=0,38$, respectivamente; $132 \mathrm{gl}$ e $104 \mathrm{gls}$, respectivamente; $\mathrm{p}<0,01$ para ambos os casos ) e inversamente correlacionada com Estar Amando ( $r=-024$ e 0,21 , respectivamente). Para os homens a quantidade de amores está relacionada com a Idade $(r=0,29$; $109 \mathrm{gl}, \mathrm{p}<0,01)$. Todas estas correlações são fracas, embora estatisticamente significantes. Notar que as pessoas diferenciam amor de paixão: (1) relatam muito mais paixões do que amores, e (2) estes dois sentimentos apresentam apenas uma fraca correlação.

\section{Percentagem de pessoas que estão amando atualmente}

Dados sobre este tipo de experiência Sozinho foram coletados apenas na amostra de 2002. A maioria dos participantes (64\%) declarou que estava amando no momento que respondeu esta pesquisa (Tabela 6). Estar amando, portanto, é um estado afetivo mais presente entre estes participantes do que não estar amando: $68,7 \%$ das mulheres e $60 \%$ dos homens declararam que estavam amando.

Tabela 6. Percentagens de homens e mulheres amando atualmente (Amostra de 2002)

\begin{tabular}{cccc}
\hline & Mulheres & Homens & MÉDIAS \\
\hline Amando & 68,7 & 60,0 & 64,0 \\
Não-amando & 31,7 & 40,0 & 36,0 \\
\hline TOTAIS & 100 & 100 & 100 \\
\hline
\end{tabular}

Estes dados estão de acordo com os aqueles relatados por Kenrick e Kenrick (1986) e por Sprecher e cols. (1994): a maioria dos pesquisados se declaram amando.

\section{Amando $X$ não-amando atualmente - relações com outros tipos de experiências amorosas}

Existe uma relação inversa entre estar amando atualmente e a quantidade de vezes que amou anteriormente tanto para as mulheres $(\mathrm{r}=-0,24,139 \mathrm{gl}, \mathrm{p}<0,05)$ como para os homens $(\mathrm{r}=-0,21 ; 108 \mathrm{gl}, \mathrm{p}<0,05)$. Esta correlação é fraca, mas indica que aqueles que amam muitas vezes têm uma maior probabilidade de não estarem amando no presente.

\section{CONCLUSÕES}

Neste artigo foram analisadas as incidências e variações de cinco tipos de situações amorosas e de seis tipos de experiências amorosas, as relações entre estas situações e experiências e o sexo dos participantes.
A grande maioria dos participantes deste estudo era constituída por "solteiros" (Só, Paquerando ou Namorando), tal como este termo é usado pelo IBGE. As incidências de Casados ou Morando com Alguém são menores neste estudo do que aquelas relatadas pelo IBGE para as categorias correspondentes. Embora o planejamento experimental do presente estudo não permita determinar as causas destas discrepâncias, parece provável que o fato dos participantes desta pesquisa serem estudantes universitários e terem um nível econômico maior do que a média da população brasileira sejam seus dois motivos.

Esta pesquisa verificou que o sexo dos participantes tem pequena relação com as incidências das situações amorosas pesquisadas, mas que teve uma influência importante na incidência de vários dos tipos de experiências amorosas pesquisados - tanto nas suas médias de incidência como nos seus padrões de variabilidade. As semelhanças nas incidências de homens e mulheres nestas situações e experiências são maiores do que suas diferenças. Um dos motivos para estas 
semelhanças é que muitas destas situações e experiências amorosas ocorrem entre um homem e uma mulher, o que uniformiza as incidências para os dois sexos.

Este estudo também procurou identificar as relações entre as experiências amorosas. Foram constatados vários tipos de relações embora todas elas sejam pequenas: a grande maioria das correlações de Pearson foram menores do que 0,40. Estas pequenas correlações são bastante comuns em estudos de tipo e provavelmente ocorrem porque tais fenômenos são multicausados (Rhode \& cols., 2005). Por exemplo, uma pessoa pode ficar ou deixar de ficar devido a uma grande quantidade de motivos. Cada um destes motivos impõe um certo grau de variabilidade à ocorrência do ficar, o que enfraquece as relações entre cada uma de suas possíveis causas e a ocorrência desta prática amorosa.

É necessária a elaboração de um modelo que explique as inter-relações entre estas experiências amorosas. Parece que algumas destas relações acontecem simplesmente porque os fenômenos são um prérequisito um para o outro ou são impostas social ou biologicamente. Por exemplo, muitas mulheres exigem um certo grau de paixão ou amor como prérequisito para o sexo. Se tais práticas são diádicas, basta um dos dois parceiros impor o pré-requisito para que a relação entre as variáveis seja constatada para ambos. Algumas destas experiências simplesmente aumentam as chances para que outras ocorram. Por exemplo, embora para namorar não seja necessário ficar, quem fica já se expôs a uma situação amorosa e cumpriu alguns dos requisitos necessários para namorar aquela pessoa que está ficando.

Outros tipos e situações e experiências amorosas devem ser incluídos em estudos futuros sobre este tema. Por exemplo, o noivado, a situação "Amantes" e o "rolo" também merecem ser estudados.

A grande quantidade de replicações dos resultados que foram verificados entre as subamostras de $2002 \mathrm{e}$ 2005 indica que estas conclusões podem ser generalizadas para outros grupos assemelhados. Por outro lado, como os participantes de ambas estas amostras eram universitários e freqüentavam faculdades da cidade de São Paulo, não é prudente generalizar tais conclusões para outros grupos como, por exemplo, aqueles constituídos por pessoas de diferentes faixas etárias, habitantes de outras partes de Brasil e não universitários.
Estes resultados atestam a forte presença do relacionamento amoroso na vida dos participantes desta pesquisa e amplia o impacto da afirmação de Gottman e Carrère (1994) de que cerca de 90\% das pessoas da humanidade se casam pelo menos uma vez. Esta pesquisa mostra que os seres humanos, além de se casarem, participam de vários outros tipos de relacionamentos e experiências amorosas.

\section{REFERÊNCIAS}

Abdo, C. H. N., Moreira Jr., E. D. \& Fittipaldi, J. A. (2000). Estudo do comportamentosexual no Brasil. ECOS. Revista Brasileira de Medicina, 57 (11), 1329-35.

Amélio, A. \& Mantinez, M. (2005). Para viver um grande amor. São Paulo: Editora Gente.

Buss, M. D. (1994). The evolution of desire. New York: Basic Books.

Buss, D. M., Abbott, M., Angleitner, A., Asherian, A., Biaggio, A., Blancovillasenor, A., Bruchonschweitzer, M., Chu, Hy, Czapinski, J., DeRaad, B., Ekehammar, B., Ellohamy, N., Fioravanti, M., Georgas, J., Gjerde, P., Guttman, R., Hazan, F., Iwawaki, S., Jana kiramaiah, N., Khosroshani, F., Kreitler, S., Lachenicht, L, Lee, M., Liik, K., Little, B., Mika, S., Moadelshahid, M., Moane, G., Montero, M., Mundycastle, A. C., Niit, T., Nsenduluka, E., Pienkowski, R., Pirttila-Backman, A. M., Deleon, J. P, Rousseau, J., Runco, M. A., Safir, M. P., Samuels, C., Sanitioso, R., Serpell, R., Smid,, N., Spencer, C., Tadinac, M., Todorova, E. N., Troland, K., Vandenbrande, L., Van Heck, G., Vanlangenhove, L. \& Yang, K. S. (1990). International preferences in selecting mates - a study of 37 cultures. Journal of Cross-Cultural Psychology, 21, 5-47.

Darwin, C. (1871). The descent of man and selection in relation to sex. London: Murray.

Gottman, J. M. \& Carrère, S. (1994). Why can't men and women get along? Developmental roots and marital inequities. Em D. J. Canary \& L. Sttaford (Orgs.), Communication and Relational Maintenance. San Diego: Academic Press, Inc.

Gottman, J. M. \& Notarius, C. I. (2002). Marital research in the $20^{\text {th }}$ century and a research agenda for the $21^{\text {th }}$ century. Family Process, 41 (2), 158-197.

Gregersen, E. (1983). Práticas sexuais: a história da sexualidade humana. São Paulo: Roca.

Hendrick, C. \& Hendrick, S. (1986). A theory and method of love. Journal of Personality and Social Psychology, 50, 392- 402.

Hite, S. (1992). O Relatório Hite. (21 a ed.). São Paulo: Bertrand Brasil.

Hunt, M. (1974). Sexo: teoria e prática. São Paulo: IBRASA.

IBGE - Instituto Brasileiro de Geografia e Estatística (2002). Pesquisa Nacional por Amostra de Domicílios - PNAD 2002 [On-line]. Retirado em 28/08/2006 do World Wide Web: http://www.ibge.gov.br/home/estatistica/populacao/trabalhoere ndimento/pnad2002/default.shtm

Kaplan, H. S. (1977). A nova terapia do dexo. Rio de Janeiro: Editora Nova Fronteira. 
Kinsey, A. C., Pomeroy, W. B. \& Martin, C. E. (1948). Sexual behavior in the human male. Philadelphia, W. B. Saunders Co.

Masters, W. H \& Johnson, V. E. (1984). A resposta sexual humana. São Paulo: Roca.

Michael, R. T., Gagnon, J. H., Laumann, E. O. \& Kolata, G. (1995). Sex in America. New York: Warner Books

Pinsof, W. M. (2002). The death of "Till death us do part": the transformation of pair-bonding in the $20^{\text {th }}$ century. Family Process, 41 (2), 135-157.

Rhodes, G., Simmons, L. W. \& Peters, M. (2005). Atrractiveness and sexual behavior: does attractiveness enhance mating success? Evolution and Human Behavior, 26, 186-201.

Seligman, M. E. P. (2002). Felicidade autêntica. Rio de Janeiro: Editora Objetiva Ltda.

Shaver, P., Hazan, C. \& Bradshaw, D. (1988). Love as attachment: the integration of three behavioral systems. Em R. J. Sternberg, M. L. Barnes (Orgs.), The Psychology of love (pp. 68-99). Birghamton: Yale University Press.

Silva, A. A. (1996, julho). Sexo casual. Viver Psicologia, 16-18.
Silva, A. A., Mayor, A. S., Almeida, T., Rodrigues, A., Oliveira, L. M. \& Martinez, M. (2005). Determinação das histórias de amor mais adequadas para descrever relacionamentos amorosos e identificação das histórias de amor que produzem mais identificação, menos identificação e que as pessoas mais gostariam de viver. Interação em Psicologia, 9 (2), 295-309.

Sprecher, S., Aron, A., Hatfield, E., Cortese, A., Potapova, E. \& Levitskaya, A. (1994). Love: American style, Russian style, and Japanese style. Personal Relationship, 1, 349-369.

Stafford, L. \& Kline, S. (2004). Married individuals, cohabiters, and cohabiters who marry: a longitudinal study of relational and individual well-being. Journal of Social and Personal Relationships, 21 (12), 231-248.

Sternberg, R. J. \& Barnes, M. L. (1988). The psychology of love. Birghamton: Yale University Press.

Recebido: 10/09/2006

Revisado: 20/12/2006 Aceito: 30/12/2006

\section{Notas:}

1 Os nomes destas variáveis serão grafados com suas iniciais em maiúsculas para diferenciá-las do uso corrente destes termos.

2 Agradeço aos seguintes alunos pela coleta dos dados e por uma parte das tabulações: Yone Xavier Felipe, Denise Bragotto, Carlos Eduardo Lacaz, Claudirene Favarin, Paulo Santana, Rosa Tedeschi, Telma Gozzi.

\section{Sobre o autor:}

Ailton Amélio da Silva: Doutor em Psicologia; psicólogo clínico; escritor; professor do Instituto de Psicologia da USP. Endereço eletrônico: ailton@uol.com.br. 